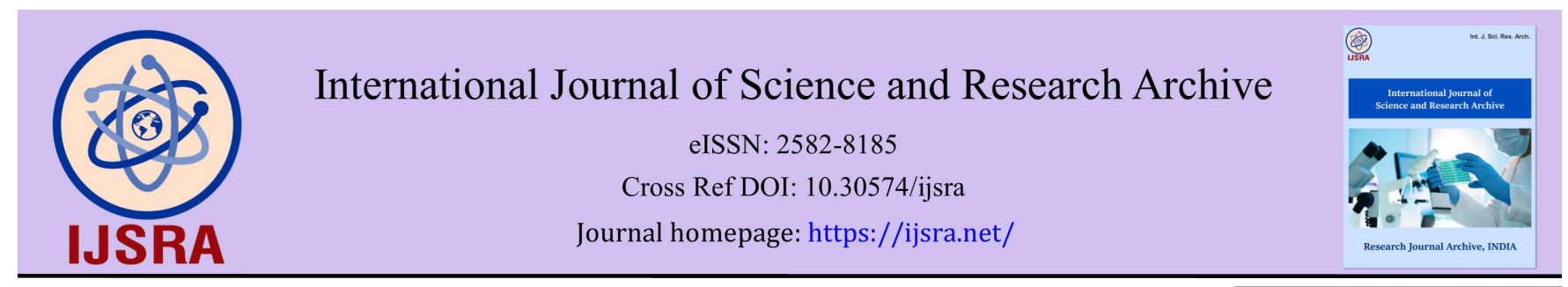

(REVIEW ARTICLE)

\title{
Acinetobacter baumannii Quorum sensing: A way to communicate and a target to eradicate
}

\author{
Elkheloui Raja, Hamadi Fatima * and Mimouni Rachida \\ Laboratory of Microbial Biotechnology and Plants Protection, Ibn Zohr University, Faculty of Sciences, Agadir-Morocco.
}

International Journal of Science and Research Archive, 2021, 03(02), 134-145

Publication history: Received on 05 July 2021; revised on 24 August 2021; accepted on 26 August 2021

Article DOI: https://doi.org/10.30574/ijsra.2021.3.2.0112

\begin{abstract}
Quorum sensing is a communication system based on the actions of chemical signal molecules depending on the density of the cell population. These molecules are widely considered as effectors of the gene expression of several virulence factors. As a result, it has attracted a lot of attention because of its possible applicability as a target for treating infections. This review attempts to give a description of this system on gram negative bacteria specifically on Acinetobacter baumannii as an important nosocomial pathogen. Additionally, quorum sensing in biofilm will be also treated because it is considered as the origin of several chronic infections. Numerous studies have been carried out to prove the role of inhibitors in the disruption of quorum sensing, known as quorum quenching. Quorum quenching is a new strategy to eradicate bacterial infections due to the crucial intervention of quorum sensing in different virulence factors and particularly in the biofilm formation.
\end{abstract}

Keywords: Quorum sensing; Acylated homoserine lactone; Biofilm; Acinetobacter baumannii; Anti-infection

\section{Introduction}

In recent decades, it has become clear that bacteria coordinate interactions with each other and with higher organisms through intercellular communication systems, called quorum sensing (QS). These latter are often based on the expression of new genes $[1,2]$. The first works highlighting the mechanism of bacterial communication appeared in 1965 and $1970[3,4]$. The researchers established a link between the control of the genetic competence of Streptococcus pneumonia [4] and the control of the activity of luminescent bacteria [3] and the production of extracellular molecules. In 1980, other important studies were published on the luminescent genes of Vibrio fischeri [5] and the autoinducer was the N-3-oxohexanoyl-L-homoserine lactone-30C6-HSL [6]. The term quorum sensing was first used by Fuqua et al (1994) in a review article. These authors defined quorum sensing as an environmental sensing self-induction system that allows bacteria to monitor their own population density, producing a diffusible compound called an autoinducer [7].

The importance of this system is reflected by its involvement in various bacterial processes and behaviors such as bioluminescence, sporulation, biofilm formation, secretion of virulence factors, symbiosis, and conjugation [8-12]. Thus, QS represents an interesting target for developing a new strategy to combat infections caused by bacterial pathogens.

Herein, we aim to provide an overview of simple and summarized information about QS system, focusing on gramnegative bacteria. It will also cover the close relationship between pathogenicity and QS, giving the example of Acinetobacter baumannii and its biofilm formation as one of the most frequent nosocomial pathogens. At the end of this review, the possible application of QS as one of the probable anti-infectious strategies will be discussed.

\footnotetext{
* Corresponding author: Fatima Hamadi

Laboratory of Microbial Biotechnology and Plants Protection, Ibn Zohr University, Faculty of Sciences,Agadir-Morocco. 


\section{Definition of Quorum sensing}

QS is a bacterial communication based on ligand-receptor signal molecules; these are so-called self-inducing bacterial pheromones secreted by bacteria to regulate certain functions [13]. Gene expression of these pheromones will only take place when a bacterial population reaches a significant cell density threshold, thus leading to the activation or repression of the genes. Therefore, any bacterial population capable of expressing these autoinducers is called a quorum $[13,14]$.

The QS mechanism is based on an enzyme that catalyzes the synthesis of chemical signals and a receptor that binds with these signals to induce the expression of genes responsible for various bacterial functions (sporulation, biofilm formation, conjugation, motility) and virulence factors (proteases, toxins, and adhesins) [15, 16]. QS is a very common molecular mechanism in bacteria, which gives them an important advantage in terms of evolution, allowing them to adapt to changes in the environment [17]. Moreover, it permits the bacteria to coexist in a community and express phenotypes that are beneficial and ensure bacterial survival [18]. The expression of autoinducers depends on the nature of the bacteria (QS used by both Gram positive and Gram negative) and its physiological state (cell density), but also requires the presence of other elements: synthase signals, receptor signals, genes and regulatory signals [19].

\section{Quorum sensing system in gram negative bacteria}

Gram negative bacteria produce autoinducers from an initial precursor called Acylated Homoserine Lactone (AHL or Nacyl homoserine lactones). AHL synthesis is catalyzed by enzymes of the signal synthase family (LuxI; enzymes encoded by the Lux operon). The transcription of the Lux operon depends on cell density; its activation requires significant cell density [19]. The system of QS is well known for several gram-negative bacterial species such as Pseudomonas aeruginosa and Agrobacterium tumefaciens. These two species are able to couple their gene expression according to fluctuations in cell density [20].

AHL is also known as AI-1 and it consists of homoserine lactone rings with an additional fatty acid side chain, which differ in length, and their residue depending on the bacterial species [18]. Even AHL signaling has traditionally been considered as intraspecific communication, V. C. Kalia (2013) suggests that AHL can also be used to detect potential environmental competitors.

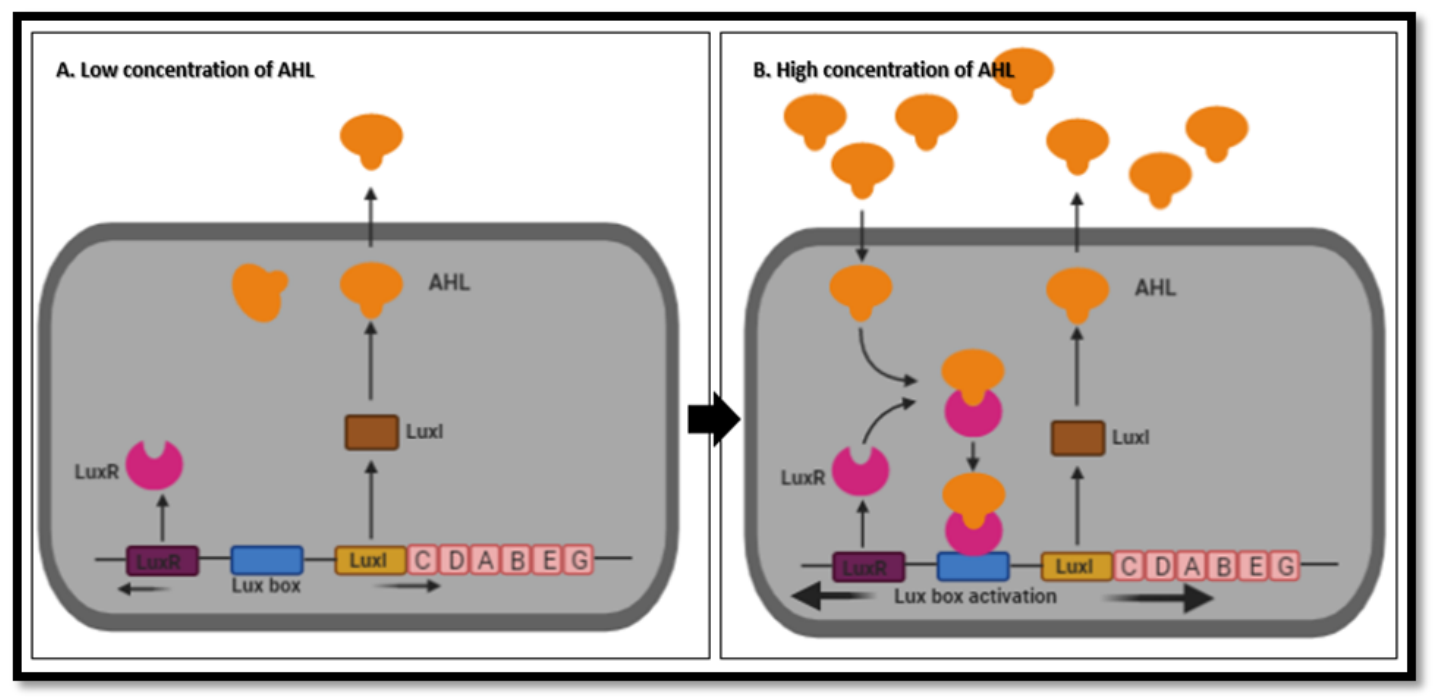

Figure 1 LuxI/LuxR QS system of Vibrio fischeri with bioluminescence regulation A: at low population density and B: high population density

In the 1980, the first LuxI/LuxR quorum detection mechanism was discovered in Vibrio fischeri (fig. 1), whose bioluminescence is controlled by the regulation of a transcriptional regulatory protein (LuxR) and a signal synthase molecule (LuxI) [5, 22]. The LuxI enzyme catalyzes the amide bond formation between the substrates Sadenosylmethionine (SAM) and the acyl-acyl carrier protein (acylACP) to form AHL signal molecule. Cells express LuxI even when the population density is low (fig. 1A), but the concentration of AHL is maintained at a low level [23, 24]. When the population density increases, the concentration of AHL in the medium gradually rises and reaches a threshold 
level (corresponding to the detection of quorum). Then, the AHL enters the cell and binds to the LuxR protein (fig. 1B). The LuxR complex that is activated by AHL binds to a lux box located at the LuxI transcription start site. The LuxICDABEG transcription is subsequently activated (fig. 1B), resulting in an increase in light output and AHL [23, 24]. The newly generated signal molecules are capable of activating more LuxR proteins, leading to self-induction, and then the exponential production of bioluminescent molecules and signals [23,24]. Since the discovery of the LuxI/LuxR quorum, many Gram-negative bacteria have been found to regulate the quorum detection system using LuxI/LuxR homologues (Table 1).

Table 1 Quorum sensing systems of Gram-negative bacteria with their regulated functions

\begin{tabular}{|c|c|l|c|}
\hline Microorganism & QS system & \multicolumn{1}{|c|}{ Regulated functions } & Reference \\
\hline Acinetobacter baumannii & Lux (Aba) & Motility and biofilm formation & {$[25]$} \\
\hline Vibrio fischeri & LuxI, Ain, LuxS & $\begin{array}{l}\text { Expression of bioluminescence, colonization in the } \\
\text { host, and motility }\end{array}$ & {$[16]$} \\
\hline Pseudomonas aeruginosa & $\begin{array}{c}\text { Las, Rhl, PQS, } \\
\text { IQS }\end{array}$ & $\begin{array}{l}\text { Virulence factors: pyocyanin, pyoverdine, elastase, } \\
\text { alkaline protease, motility, rhamnolipids, and } \\
\text { biofilm formation }\end{array}$ & {$[2]$} \\
\hline Escherichia coli & SdiA & Motility and biofilm formation & {$[26]$} \\
\hline Serratia liquefaciens & Swr & Motility, biofilm formation & {$[27]$} \\
\hline
\end{tabular}

\section{Others Quorum sensing systems}

The third class of signaling molecules is the autoinducer 2 (AI-2), which can be found in Gram-positive and Gramnegative bacteria. The AI-2 system was first described in Vibrio harveyi and its extracellular signaling molecule is a furanosyl borate diester [28].This class is widely involved in interspecies communication (cross talk), and is therefore considered a universal communication signal between different bacterial species [29].

Quinolone, the PQS (Pseudomonas Quinolone Signal) autoinducer, also known as quinolone, identified in Pseudomonas aeruginosa (table1), is produced by proteins encoded by the pqsABCDH genes. Together with other AHL autoinducers, PQS controls biofilm formation and the production of virulence factors such as elastase, pyocyanin and leticin [30, 31].

Recently, the autoinducer IQS (2-(2-hydroxyphenyl)-thiazole-4-carbaldehyde) was found in Pseudomonas aeruginosa (table1), it is produced by proteins encoded by AmbBCDE. This molecule controls the expression of several genes associated with the production of pyocyanin, rhamnolipids and elastase in response to phosphate-stress [2].

\section{Quorum sensing and pathogenicity}

Several studies have shown that cell-to-cell communication and regulation of numerous virulence factors in bacteria are managed by QS systems [32-36]. In Staphylococcus aureus, the Agr system controls the production and dissemination of biofilm, consequently, inducing both acute and chronic biofilm-associated infections [36]. Moreover, Arg is involved in the production of many toxins and degradable exoenzymes in Staphylococcus aureus [34]. For Pseudomonas aeruginosa, AHL controls approximately 300 genes responsible for various cellular functions, including its pathogenesis in response to the surrounding environment [37]. The LasI is one of the major mechanisms of pathogenicity and resistance of Pseudomonas aeruginosa. It regulates the biofilm formation, and virulence factors such as exotoxin A, elastase, pyocyanin, alkaline phosphatase, and rhamnolipid [35]. Similarly, Bacillus cereus virulence factors, such as phospholipases C, proteases and hemolysins, are controlled by the expression of QS system [38]. QS also contributes to the expression of several genes of virulence and survival within the host cell in Brucella melitensis [39]. It is becoming a fact that the biofilm is a protective barrier against the external environments. It gives to the bacteria the ability to maintain communities efficiently by secreting extracellular molecules (QS molecules) to communicate [4042]. Moreover, biofilm allows microorganisms to be more resistant to the antimicrobial treatments by preventing the spread of antimicrobial agents and expression of gene involved in resistance [43]. Interestingly, when bacterial population densities are low, the expression of virulence genes is disactivated to avoid detection of the pathogen and immune stimulation against pathogenicity factors. This gives the bacteria sufficient time to colonize and establish themselves in the host. Once established and having reached a sufficient number, the pathogen begins its virulence activities and causes a total infection [11]. 


\section{Acinetobacter baumannii quorum sensing}

The genus Acinetobacter comprises a group of non-motile bacteria, Gram-negative coccobacilli, with strict aerobic metabolism [44]. They are catalase-positive, oxidase-negative and grow well at an incubation temperature of $37^{\circ} \mathrm{C}$ [45]. A. baumannii is an important nosocomial opportunistic human pathogen that is gradually gaining more attention as a major health threat worldwide. Over the last 6 years, the number of deaths caused by drug resistant A. baumannii has increased by approximately $60 \%$ [46]. This bacterium has a remarkable ability to upregulate and acquire the determinants of antibiotic resistance and to form biofilm [47-50].

Table 2 Examples of A. baumannii Acylated homoserine lactones [51].

\begin{tabular}{|c|c|c|}
\hline $\begin{array}{l}\text { Acyl Homoserine } \\
\text { Lactones }\end{array}$ & $\begin{array}{l}\text { Molecular } \\
\text { Formula }\end{array}$ & Structure \\
\hline $\begin{array}{l}\mathrm{N}-(3- \\
\text { hydroxydodecanoyl) } \\
\text {-L-homoserine } \\
\text { lactone } \\
\text { (3-OH-C12-HSL) }\end{array}$ & $\begin{array}{l}\mathrm{C} 16 \mathrm{H} 29 \mathrm{NO} \\
4\end{array}$ & \\
\hline $\begin{array}{l}\text { N-Dodecanoyl-L- } \\
\text { homoserine lactone } \\
\text { (Unsubstituted C-12- } \\
\text { HSL) }\end{array}$ & $\begin{array}{l}\mathrm{C} 16 \mathrm{H} 29 \mathrm{NO} \\
3\end{array}$ & \\
\hline $\begin{array}{l}\text { N-Dodecanoyl-L- } \\
\text { homoserine lactone } \\
\text { (Unsubstituted C-10- } \\
\text { HSL) }\end{array}$ & $\begin{array}{l}\mathrm{C} 14 \mathrm{H} 25 \mathrm{NO} \\
3\end{array}$ & \\
\hline $\begin{array}{l}\text { N-Tetradecanoyl-L- } \\
\text { homoserine lactone } \\
\text { (Unsaturated C-14- } \\
\text { HSL) }\end{array}$ & $\begin{array}{l}\text { C18H33NO } \\
3\end{array}$ & \\
\hline $\begin{array}{l}\mathrm{N}-(3- \\
\text { Oxotridecanoyl)-L- } \\
\text { homoserine lactone } \\
\text { (Unsaturated 3-oxo- } \\
\text { C13-HSL) }\end{array}$ & $\begin{array}{l}\mathrm{C} 17 \mathrm{H} 29 \mathrm{NO} \\
4\end{array}$ & \\
\hline $\begin{array}{l}\mathrm{N}- \\
\text { Hexanoyldecanoyl-L- } \\
\text { homoserine lactone } \\
\text { (Unsaturated C-16- } \\
\text { HSL) }\end{array}$ & $\begin{array}{l}\mathrm{C} 20 \mathrm{H} 35 \mathrm{NO} \\
3\end{array}$ & \\
\hline
\end{tabular}

A. baumannii QS machinery is mediated by a two-component system which is homologous to the typical LuxI/LuxR system found in Gram-negative bacteria, Abal/AbaR genes. These genes were acquired horizontally from Halothiobacillus neapolitanus [51]. The AbaI belongs to LuxI family of autoinducer synthases that produced more than one AHL (table 2) for 63\% of Acinetobacter strains [51]. For example, AbaI gene of A. baumannii strain M2 has been cloned in Esherichia coli which catalyzed the synthesis of N-(3-hydroxydodecanoyl)-L-HSL (3-hydroxy-C12-HSL) [51]. The 3-hydroxy-C12-HSL was the primary AHL of $A$. baumannii and minor amounts of additional AHLs were also identified [52]. Subsequently, the autoinducer receptor protein AbaR interacts with AHL to control the gene expression 
of A. baumannii [51]. Another study demonstrated that QS signaling molecules enhance expression of the chaperoneusher secretion system (which is necessary for the motility of contractions), allowing A. baumannii to easily attach to abiotic surfaces and to form biofilms [53]. According to antibiotic resistance, an AHL-deficient A. baumannii strain S mutant (AbS-M) was more sensitive to meropenem and piperacillin than wild-type AbS [25]. When AHL N-3-hydroxydodecanoyl-homoserine lactone (N-3-OH-C12-HSL) was supplemented, the resistance was restored [25]. Furthermore, the drug-resistance genes were expressed by lower levels in meropenem-treated AbS-M, while the treatment with N-3OH-C12-HSL restored the expression of these genes [25].

\section{Acinetobacter baumannii biofilm and quorum sensing}

A. baumannii is widely distributed in nature and able to adhere to and colonize biotic and abiotic surfaces $[47,54,55]$. These characteristics lead the bacteria to persist and form biofilms in various hospital settings, causing widespread infections. The infection with $A$. baumannii is a cause of clinical illnesses such as pneumonia, sepsis, secondary meningitis, urinary tract infections and surgical wounds, especially in immunocompromised patients in intensive care units [56-59]. It is relevant to point out that most of infectious diseases, at least $65 \%$ are linked to bacterial communities that proliferate by forming biofilms [60]. Biofilm is a sessile community of microbial origin characterized by cells that are irreversibly attached to a substrate or an interface or to each other. These attached cells are ensconced in a matrix of extracellular polymeric substances produced by them, and exhibit an altered phenotype with regard to growth rate and gene transcription [61]. It is estimated that 60 to $80 \%$ of bacterial infections are caused by biofilms [62]. Efforts to disrupt biofilms have enabled to target QS as one of the implicated factors on biofilm formation. Recent studies have linked biofilm development to QS [63-65]. In this regard, maturation of A. baumannii biofilm needs communication among bacteria with respect to cell density $[52,66]$. Moreover, the mutation in AbaI resulted in $30-40 \%$ reduction in biofilm when compared to no mutant strain [67]. Suppression of AbaI reduces biofilm formation in A. baumannii and exogenous addition of purified AHL restored it [52]. In addition, the loss of AbaI activity results in reduced survival and growth of soft tissue infections [68]. In addition, an AHL synthase-deficient A. baumannii displayed a reduced capacity to form biofilm compared to its wild one [69].

Aba QS system plays an essential role in A. baumannii by regulating virulence factors, biofilm formation, surface motility and bacterial competence [70]. To evaluate the effects of abaR on abal expression, motility, biofilm and pellicle formation by A. baumannii, Oh and Han (2020) have constructed an isogenic mutant of abaR. The mutant resulted in a decreased abaI expression, substantial defects in motility and biofilm and pellicle formation by this pathogen [71]. In addition, a study based on RNAseq showed that 264 genes were upregulated in A. baumannii biofilms [72]. Alcohol dehydrogenase encoding gene, a QS molecule that plays a key role in A. baumannii biofilm formation, was one of the most upregulated genes [72].

\section{Quorum quenching}

The increasing bacterial resistance and biofilm formation ability make the fight of bacterial infections more complicated, thus the development of new antimicrobial alternatives is needed. As mentioned before, QS plays a crucial role in various bacterial functions such as pathogenicity, biofilm formation, expression of virulence factors, and antibiotic resistance. Consequently, QS can be one of the effective therapeutic targets, technically referred to as "Quorum Quenching QQ" or quorum sensing inhibitors (QSI) [15, 16, 73, 74]. The properties of QQ (chemical compounds, enzymes), the mechanisms of action (inhibition, competition, prohibition of QS signal, etc.) and the targets are different. Consequently, the dysfunction of the QS system can be achieved by different methods: 1 reducing the activity of the AHL-related receptor protein or AHL synthase, 2 inhibiting the production of QS signaling molecules, 3 degrading AHL, and 4 mimicking signaling molecules using mainly synthetic compounds as analogues of signaling molecules [21]. The first halogenated furanone QQ compound that interferes with bacterial QS was identified from the Australian marine red alga Delisea pulchra [75].

Since antiquity, plants and their derivatives have been used in the treatment of diseases [76, 77]. This is due to their extremely complex composition, containing several compounds such as terpenoids, acids, alcohols, aldehydes, aliphatic hydrocarbons and acyclic esters [77]. Therefore, numerous studies have been carried out in this framework, to identify new herbal agents that could have a QQ effect. The QQ activity of Cinnamon oil against P. aeruginosa was investigated by testing the inhibition of biofilm formation and other virulence factors such as pyocyanin, rhamnolipid, protease, alginate production, and swarming activity [78]. The results have showed that Cinnamon oil can influence QS-based mechanisms in P. aeruginosa PA01[78]. The phenolic extract of wild strawberry (Rubus rosaefolius) was tested for its QQ activity against Chromobacterium violaceum ATCC6357, Aeromona hydrophila I0C/FDA110-36 and Serratia marcescens UFOP-001 [79]. It has been proven that the extract was able to inhibite all the phenotypes typically regulated 
by quorum sensing in bacteria, including violacein production, swarming motility and biofilm formation [79]. Another work on the anti-QS and antibiofilm effects of Thymus daenensis and Satureja hortensis essential oils against Staphylococcus aureus showed that these oils have an anti-QS effect by inhibiting the formation of Staphylococcus aureus biofilm [80]. By the same token, Syzygium aromaticum, Dionysia revolute and Eucalyptus camaldulensis showed anti-QS activities through reducing the violacein formation depletion of QS signals produced by Aeromonas veronii and Pseudomonas aeruginosa [81]. In addition, Aqueous extracts of edible plants and fruits such as Ananas comosus, Musa paradiciaca, Manilkara zapota and Ocimum sanctum have been shown to significantly reduce AHL-mediated violacein production in Chromobacterium violaceum as well as pyocyanin pigment, staphylolytic protease, elastase production and biofilm formation in Pseudomonas aeruginosa PA01 [82].

Naturally occurring QQ activities have been reported for a large number of organisms. But in some cases, the main limitation of these QS inhibitors is the low concentration in which they are produced and the associated toxicity [21]. We can circumvent these limitations by chemically synthesizing them [21]. In the same context, the condensation of anthranilate and $\beta$-keto fatty acids such as ( $\beta$-ketodecanoic acid) results in the production of secondary metabolites such as 2-heptyl-3-hydroxy-4-quinolone (the Pseudomonas quinolone signal) [21]. A methyl anthranilate (an anthranilate analogue) suppressed the production of Pseudomonas quinolone signal and reduced elastase production without affecting the growth of Pseudomonas aeruginosa PA01[21]. Similary, the bioluminescence in Vibrio fischeri was inhibited by AHLs carrying various substitutions at the C4 position of the acyl chain of 30C6HSL or C6HSL [83].

QQ molecules have several advantages compared to others antimicrobial molecules: lesser selective pressure than antibiotics, minimal impact on host commensal flora, inactivate the target rapidly, supplementation of antibiotics to increase efficacy, protection for immunocompromised and unvaccinated individuals, and block the secretion of multiple virulence factors [84]. Furthermore, comparing QQ method to antibiotics which mainly aim at inhibiting and killing microorganisms, targeting QS is widely accepted as an antivirulence (attenuate bacterial virulence) strategy as it is nonbactericidal. Therefore, it doesn't increase the antibiotic resistance of strains [85].

\section{Anti-Acinetobacter baumannii quorum sensing}

In 2017, the World Health Organization published a catalog of 12 bacteria (grouped into three levels; critical, high and medium) that urgently require the development of new antimicrobial agents. Among these species, carbapenemresistant $A$. baumannii is considered as one of the most redoubtable species (critical level) [86]. Previous research demonstrated that $A$. baumannii possessed a strong ability to form biofilms and rapidly develop antibiotic resistance [87-89]. This made it difficult for clinicians and healthcare providers to treat and control its spread leading to death.

QS signaling and biofilm formation have been positively associated with the development of microbial resistance, virulence, and the spread of resistance $[33,90]$. Moreover, Biofilm formation depends on the cell density at which cells coordinate via QS molecules, resulting in biofilm formation [91]. The formation of biofilm by this bacterium depends on AHLs of which 3-hydroxy-C12-homoserine lactone is the most predominant [65]. Hence, targeting the QS system could accelerate the development of effective intervention strategies [65]. Meanwhile, several attempts have been performed to interfere with QS as an effective target against A. baumannii (table 3) [69, 92-99] .

Luís et al (2016) carried out a study based on the analysis of the antioxidant, antibacterial and anti-quorum sensing activities of the essential oils of Eucalyptus globulus and Eucalyptus radiata. They have found that both oils have an ability to inhibit QS, by inhibiting the production of violet pigments regulated by QS in A. baumannii without interfering with their growth [93]. An interesting work has synthesized 22 inhibitors of quorum detection by mimicking the structure of the autoinducer and acinetobactin of $A$. baumannii [100]. The partially purified fraction of Glycyrrhiza glabra down-regulated the expression of the autoinducing synthase gene abal of $A$. baumannii. It has therefore reduced (92\%) the production of 3-OH-C12-HSL (A. baumannii AHL) and then regulated virulence factors (motility, biofilm formation and antioxidant enzymes production) [94]. Essential oils of Cinnamomum verum, Thymus vulgaris and Eugenia caryophyllata have a remarkable anti-biofilm and anti-QS activities against all tested species which $A$. baumannii is among them [95]. Additionally, Linalool, the main compound of Coriandrum sativum, have the capacity to interfere with the QS, to alter the adhesion to surfaces, to inhibit the biofilm formation and to disperse the established biofilms of A. baumannii [92]. Besides, a modified AHLase, as a QQ enzyme, could disrupt biofilm formation in a clinical isolate of A. baumannii S1 [69]. Pentacyclic triterpenoids could disrupt A. baumannii AHL-based signaling, thus leading to inhibition of biofilm formation and destabilization of the overall structure of biofilms [96]. Results from Mayer et al (2020) demonstrated that the combination of QQ strategies and other enzyme treatments such as DNase could represent a fairly effective approach to prevent the colonization of $A$. baumannii on surfaces and therefore also prevent infections caused by this pathogen. A recent study found that a marine steroid, Siphonocholine (Syph-1), isolated from Siphonochalina siphonella, can inhibit biofilm formation in A. baumannii and has anti-QS properties [98]. 
Regarding antibiotics, Saroj and Rather (2013) have found that Streptomycin at the subinhibitory concentration had a potential QQ activity against $A$. baumannii. they suggested that the subminimal inhibitory concentration of Streptomycin may act as an antagonist of 3-OH-C12-HSL, interfering with the binding of the signal to the AbaR protein [99].

Interestingly, Acinetobacter has been cited among few bacteria known to possess both AHL-producing and -degrading activities (producing QQ molecules) [21,101].

Table 3 Examples of quorum quenching strategies against $A$. baumannii

\begin{tabular}{|c|c|c|c|}
\hline Inhibitor & Source & Activity & Reference \\
\hline Essential oils & $\begin{array}{l}\text { Eucalyptus globulus } \\
\text { Eucalyptus radiata }\end{array}$ & $\begin{array}{l}\text { Inhibition of the production of violet } \\
\text { pigments regulated by QS in } A \text {. baumannii }\end{array}$ & [93] \\
\hline $\begin{array}{l}\text { Partially purified } \\
\text { fraction }\end{array}$ & Glycyrrhiza glabra & $\begin{array}{l}\text { Down-regulation of the expression of the } \\
\text { autoinducing synthase gene abaI }\end{array}$ & [94] \\
\hline Essential oils & $\begin{array}{l}\text { Cinnamomum verum } \\
\text { Thymus vulgaris } \\
\text { Eugenia caryophyllata }\end{array}$ & Anti-biofilm and anti-QS activities & [95] \\
\hline Linalool & Coriandrum sativum & $\begin{array}{l}\text { Interfering with the QS, to alter the adhesion } \\
\text { to surfaces, to inhibit the biofilm formation } \\
\text { and to disperse the established biofilms of } A \text {. } \\
\text { baumannii }\end{array}$ & [92] \\
\hline Modified AHLase & $\begin{array}{l}\text { QQ lactonase obtained by } \\
\text { directed evolution }\end{array}$ & QQ and disruption of biofilm formation & [69] \\
\hline $\begin{array}{l}\text { Pentacyclic } \\
\text { triterpenoids }\end{array}$ & - & Disruption A. baumannii AHL-based signaling & [96] \\
\hline QQ enzyme Aii20J & - & $\begin{array}{l}\text { QQ activity } \\
\text { Interfering with motility } \\
\text { Prevention of the colonization on surfaces }\end{array}$ & [97] \\
\hline Siphonocholine & Siphonochalina siphonella & $\begin{array}{l}\text { Anti-QS properties } \\
\text { Inhibition of biofilm formation }\end{array}$ & [98] \\
\hline Streptomycin & Antibiotic & Antagonist of 3-OH-C12-HSL & [99] \\
\hline
\end{tabular}

\section{Conclusion}

Bacteria do not live in solitary and secluded places, but rather communicate using a variety of chemical languages; QS system or bacterial talking. This review describes communication in gram-negative bacteria and $A$. baumannii in particular as one of the most troublesome pathogens. Since cell-cell communication controls the virulence factors of this bacteria, anti-quorum sensing tactics can be an effective target for preventing and treating infections. Biofilm and quorum detection are two sides of the same coin in the fight against bacterial infections. They are considered the hot factors that the bacteria dynamically establish during host infection or abiotic surface contamination. As a result, the implementation of antimicrobial strategies that target QS is of increasing interest, so further work to better understand these chemical languages could have enormous practical applications.

\section{Compliance with ethical standards}

\section{Acknowledgments}

The authors thank Pr Elmostafa Hamadi for English correction. 


\section{Disclosure of conflict of interest}

No conflict of interest was declared by the authors.

\section{References}

[1] Trosko JE. Evolution of microbial quorum sensing to human global quorum sensing: An insight into how gap junctional intercellular communication might be linked to the global metabolic disease crisis. Biology (Basel). 2016.

[2] Lee J, Wu J, Deng Y et al. A cell-cell communication signal integrates quorum sensing and stress response. Nat. Chem. Biol. 2013; 9(5): 339-343.

[3] Nealson KH, Platt T, Hastings JW. Cellular control of the synthesis and activity of the bacterial luminescent system. J. Bacteriol. 1970; 104(1): 313-322.

[4] Datta N, Kontomichalou P. (C) 1965 Nature Publishing Group. Nat. Publ. Gr. 1965; 208(5007): 239-241.

[5] Engebrecht JA, Silverman M. Identification of genes and gene products necessary for bacterial bioluminescence. Proc. Natl. Acad. Sci. U. S. A. 1984; 81(13 I): 4154-4158.

[6] Eberhard A, Burlingame AL, Eberhard C, et al. Structural Identification of Autoinducer of Photobacterium fischeri Luciferase. Biochemistry. 1981; 20(9): 2444-2449.

[7] Fuqua WC, Winans SC, Greenberg EP. Quorum sensing in bacteria: The LuxR-LuxI family of cell densityresponsive transcriptional regulators. J. Bacteriol. 1994; 176(2): 269-275.

[8] Williams P, Cámara M. Quorum sensing and environmental adaptation in Pseudomonas aeruginosa: a tale of regulatory networks and multifunctional signal molecules. Curr. Opin. Microbiol. 2009; 12(2): 182-191.

[9] Novick RP, Geisinger E. Quorum sensing in staphylococci. Annu. Rev. Genet. 2008; 42(December): 541-564.

[10] Ng WL, Bassler BL. Bacterial quorum-sensing network architectures. Annu. Rev. Genet. 2009; 43: 197-222.

[11] K. Bhardwaj A, Vinothkumar K, Rajpara N. Bacterial Quorum Sensing Inhibitors: Attractive Alternatives for Control of Infectious Pathogens Showing Multiple Drug Resistance. Recent Pat. Antiinfect. Drug Discov. 2013; 8(1): 68-83.

[12] El-Shaymaa A-S, Miyoshi S, Abdelaziz E. Regulation of Vibrio mimicus metalloprotease (VMP) production by the quorum-sensing master regulatory protein, LuxR. J. Environ. Conserv. Eng. 2003; 32(7): 509-509.

[13] Bassler BL. Small talk: cell-to-cell communication in bacteria. Cell. 2002; 109(4): 421-424.

[14] Xu GM. Relationships between the regulatory systems of quorum sensing and multidrug resistance. Front. Microbiol. 2016; 7(JUN): 1-6.

[15] Goswami J. Quorum Sensing by Super Bugs and their Resistance to Antibiotics, a Short Review. JuniperPublishers. 2017; 3(3): 1-7.

[16] Castillo-Juárez I, Maeda T, Mandujano-Tinoco EA, et al. Role of quorum sensing in bacterial infections. World J. Clin. Cases. 2015; 3(7): 575.

[17] Antonioli L, Blandizzi C, Pacher P, et al. Rethinking Communication in the Immune System: The Quorum Sensing Concept. Trends Immunol. 2019; 40(2): 88-97.

[18] Reuter K, Steinbach A, Helms V. Interfering with bacterial quorum sensing. Perspect. Medicin. Chem. 2016; 8: 115.

[19] Reading NC, Sperandio V. Quorum sensing: The many languages of bacteria. FEMS Microbiol. Lett. 2006; 254(1): $1-11$.

[20] Rasamiravaka T, El Jaziri M. Quorum-Sensing Mechanisms and Bacterial Response to Antibiotics in P. aeruginosa. Curr. Microbiol. 2016; 73(5): 747-753.

[21] Kalia VC. Quorum sensing inhibitors: An overview. Biotechnol. Adv. 2013; 31(2): 224-245.

[22] Dunlap PV, Greenberg EP. Control of vibrio fischeri luminescence gene expression in Escherichia coli by cyclic AMP and cyclic AMP receptor protein. J. Bacteriol. 1985; 164(1): 45-50. 
[23] Bassler BL, Wright M, Silverman MR. Multiple signalling systems controlling expression of luminescence in Vibrio harveyi: sequence and function of genes encoding a second sensory pathway. Mol. Microbiol. 1994; 13(2): 273286.

[24] Egland KA, Greenberg EP. Quorum sensing in Vibrio fischeri: Elements of the luxl promoter. Mol. Microbiol. 1999; 31(4): 1197-1204.

[25] Dou Y, Song F, Guo F et al. Acinetobacter baumannii Quorum-sensing signalling molecule induces the expression of drug-resistance genes. Mol. Med. Rep. 2017; 15(6): 4061-4068.

[26] Rossi E, Cimdins A, Lüthje P, et al. It's a gut feeling-Escherichia coli biofilm formation in the gastrointestinal tract environment. Crit. Rev. Microbiol. 2018; 44(1): 1-30.

[27] Labbate M, Queck SY, Koh KS, et al. Quorum Sensing-Controlled Biofilm Development in Serratia liquefaciens MG1. J. Bacteriol. 2004; 186(3): 692-698.

[28] Chen X, Schauder S, Potier N, et al. Structural identification of a bacterial quorum-sensing signal containing boron. Nature 2002; 415(6871): 545-549.

[29] Turan NB, Chormey DS, Büyükpınar Ç, et al. Quorum sensing: Little talks for an effective bacterial coordination. TrAC - Trends Anal. Chem. 2017; 91: 1-11.

[30] Hodgkinson JT, Gross J, Baker YR, et al. A new Pseudomonas quinolone signal (PQS) binding partner: MexG. Chem. Sci. 2016; 7(4): 2553-2562.

[31] Papenfort K, Bassler BL. Quorum sensing signal-response systems in Gram-negative bacteria. Nat. Rev. Microbiol. 2016; 14(9): 576-588.

[32] Rutherford ST, Bassler BL. Bacterial quorum sensing: Its role in virulence and possibilities for its control. Cold Spring Harb. Perspect. Med. 2012; 2(11): 1-26.

[33] Jiang Q, Chen J, Yang C et al. Quorum Sensing: A Prospective Therapeutic Target for Bacterial Diseases. Biomed Res. Int. 2019.

[34] Martin MJ, Clare S, Goulding D, et al. The agr locus regulates virulence and colonization genes in clostridium difficile 027. J. Bacteriol. 2013; 195(16): 3672-3681.

[35] Rezaie P, Pourhajibagher M, Chiniforush N, et al. The Effect of Quorum-Sensing and Efflux Pumps Interactions in Pseudomonas aeruginosa Against Photooxidative Stress. J. Lasers Med. Sci. 2018; 9(3): 161-167.

[36] Le KY, Dastgheyb S, Ho TV, Otto M. Molecular determinants of staphylococcal biofilm dispersal and structuring. Front. Cell. Infect. Microbiol. 2014; 4(NOV): 1-7.

[37] Brindhadevi K, LewisOscar F, Mylonakis E, et al. Biofilm and Quorum sensing mediated pathogenicity in Pseudomonas aeruginosa. Process Biochem. 2020; 96(September 2019): 49-57.

[38] Slamti L, Lereclus D. A cell-cell signaling peptide activates the PlcR virulence regulon in bacteria of the Bacillus cereus group. EMBO J. 2002; 21(17): 4550-4559.

[39] Weeks JN, Galindo CL, Drake KL, et al. Brucella melitensis VjbR and C12-HSL regulons: Contributions of the Ndodecanoyl homoserine lactone signaling molecule and LuxR homologue VjbR to gene expression. BMC Microbiol. 2010.

[40] Davey ME, O’toole GA. Microbial Biofilms: from Ecology to Molecular Genetics. Microbiol. Mol. Biol. Rev. 2000; 64(4): 847-867.

[41] Li YH, Tian X. Quorum sensing and bacterial social interactions in biofilms. Sensors. 2012; 12(3): $2519-2538$.

[42] Parsek MR, Greenberg EP. Sociomicrobiology: The connections between quorum sensing and biofilms. Trends Microbiol. 2005; 13(1): 27-33.

[43] Parastan R, Kargar M, Solhjoo K, Kafilzadeh F. Staphylococcus aureus biofilms: Structures, antibiotic resistance, inhibition, and vaccines. Gene Reports. 2020.

[44] Pour NK, Dusane DH, Dhakephalkar PK, et al. Biofilm formation by Acinetobacter baumanniistrains isolated from urinary tract infection and urinary catheters. FEMS Immunol. Med. Microbiol. 2011; 62(3): 328-338.

[45] Peleg AY, Seifert H, Paterson DL. Acinetobacter baumannii: emergence of a successful pathogen. Clin. Microbiol. Rev. 2008; 21(3): 538-582. 
[46] Solomon SL, Oliver KB. Antibiotic resistance threats in the United States: Stepping back from the brink. Am. Fam. Physician. 2014; 89(12): 938.

[47] Tomaras AP, Dorsey CW, Edelmann RE, Actis LA. Attachment to and biofilm formation on abiotic surfaces by Acinetobacter baumannii: Involvement of a novel chaperone-usher pili assembly system. Microbiology. 2003; 149(12): 3473-3484.

[48] Cevahir N, Demir M, Kaleli I, et al. Evaluation of biofilm production, gelatinase activity, and mannose-resistant hemagglutination in Acinetobacter baumannii strains. J Microbiol Immunol Infect. 2008; 41(6): 513-518.

[49] Bocanegra-Ibarias P, Peña-López C, Camacho-Ortiz A, et al. Genetic characterisation of drug resistance and clonal dynamics of Acinetobacter baumannii in a hospital setting in Mexico. Int. J. Antimicrob. Agents. 2015; 45(3): 309313.

[50] El-Shazly S, Dashti A, Vali L, et al. Molecular epidemiology and characterization of multiple drug-resistant (MDR) clinical isolates of Acinetobacter baumannii. Int. J. Infect. Dis. 2015; 41: 42-49.

[51] Bhargava N, Sharma P, Capalash N. Quorum sensing in Acinetobacter: An emerging pathogen. Crit. Rev. Microbiol. 2010; 36(4): 349-360.

[52] Niu C, Clemmer KM, Bonomo RA, Rather PN. Isolation and characterization of an autoinducer synthase from Acinetobacter baumannii. J. Bacteriol. 2008; 190(9): 3386-3392.

[53] Luo L, Wu L, Xiao Y, et al. Enhancing pili assembly and biofilm formation in Acinetobacter baumannii ATCC19606 using non-native acyl-homoserine lactones. BMC Microbiol. 2015; 15(1): 62.

[54] Lee JC, Koerten H, Van den Broek P, et al. Adherence of Acinetobacter baumannii strains to human bronchial epithelial cells. Res. Microbiol. 2006; 157(4): 360-366.

[55] Gospodarek E, Grzanka A, Dudziak Z, Domaniewski J. Electron-microscopic observation of adherence of Acinetobacter baumannii to red blood cells. Acta Microbiol. Pol. 1998; 47(2): 213-217.

[56] John J, Saranathan R, Adigopula LN, et al. The quorum sensing molecule N-acyl homoserine lactone produced by Acinetobacter baumannii displays antibacterial and anticancer properties. Biofouling. 2016; 32(9): 1029-1047.

[57] Sahu PK, Iyer PS, Barage SH, et al. Characterization of the algC gene expression pattern in the multidrug resistant Acinetobacter baumannii AIIMS 7 and correlation with biofilm development on abiotic surface. Sci. World J. 2014.

[58] Subhadra B, Hwan Oh M, Hee Choi C. Quorum sensing in Acinetobacter: with special emphasis on antibiotic resistance, biofilm formation and quorum quenching. AIMS Microbiol. 2016; 2(1): 27-41.

[59] Alves S, Duarte A, Sousa S, Domingues FC. Study of the major essential oil compounds of Coriandrum sativum against Acinetobacter baumannii and the effect of linalool on adhesion, biofilms and quorum sensing. Biofouling. 2016; 32(2): 155-165.

[60] Lewis K. Persister cells, dormancy and infectious disease. Nat. Rev. Microbiol. 2007; 5(1): 48-56.

[61] Donlan RM, Costerton JW. Biofilms: survival mechanisms of clinically relevant microorganisms. Clin. Microbiol. Rev. 2002; 15(2): 167-193.

[62] Report C, Ramos-gallardo G. Chronic Wounds in Burn Injury : A Case Report on Importance of Biofilms. 2016; $175-180$.

[63] Kröger C, Kary SC, Schauer K, Cameron ADS. Genetic Regulation of Virulence and Antibiotic Resistance in Acinetobacter baumannii. 2017.

[64] Paluch E, Rewak-Soroczyńska J, Jędrusik I, et al. Prevention of biofilm formation by quorum quenching. 2020.

[65] Saipriya K, Swathi CH, Ratnakar KS, Sritharan V. Quorum-sensing system in Acinetobacter baumannii: a potential target for new drug development. J. Appl. Microbiol. 2020; 128(1): 15-27.

[66] Irie Y, Parsek MR. Quorum sensing and microbial biofilms. Curr. Top. Microbiol. Immunol. 2008; 322: 67-84.

[67] Gaddy JA, Actis LA. Regulation of Acinetobacter baumannii biofilm formation. Future Microbiol. 2009; 4(3): 273278.

[68] Mayer C, Muras A, Romero M, et al. Multiple quorum quenching enzymes are active in the nosocomial pathogen Acinetobacter baumannii ATCC17978. Front. Cell. Infect. Microbiol. 2018; 8(SEP): 1-16. 
[69] Chow JY, Yang Y, Tay SB et al. Disruption of biofilm formation by the human pathogen Acinetobacter baumannii using engineered quorum-quenching lactonases. Antimicrob. Agents Chemother. 2014; 58(3): 1802-1805.

[70] Pourhajibagher M, Boluki E, Chiniforush N et al. Modulation of virulence in Acinetobacter baumannii cells surviving photodynamic treatment with toluidine blue. Photodiagnosis Photodyn. Ther. 2016; 15: $202-212$.

[71] Oh MH, Han K. AbaR is a LuxR type regulator essential for motility and the formation of biofilm and pellicle in Acinetobacter baumannii. Genes and Genomics. 2020; 42(11): 1339-1346.

[72] Zhang K, Yang X, Yang J. et al. Microbial Pathogenesis Alcohol dehydrogenase modulates quorum sensing in biofilm formations of Acinetobacter baumannii. Microb. Pathog. 2020; 148(May): 104451.

[73] García-Contreras R. Is quorum sensing interference a viable alternative to treat Pseudomonas aeruginosa infections? Front. Microbiol. 2016; 7(SEP): 1-7.

[74] Santhakumari S, Ravi AV. Targeting quorum sensing mechanism: An alternative anti-virulent strategy for the treatment of bacterial infections. South African J. Bot. 2019; 120: 81-86.

[75] Manefield M, Harris L, Rice SA, et al. Inhibition of Luminescence and Virulence in the Black Tiger Prawn (Penaeus monodon) Pathogen Vibrio harveyi by Intercellular Signal Antagonists. 2000.

[76] Rehman R, Hanif MA, Mushtaq Z, Al-Sadi AM. Biosynthesis of essential oils in aromatic plants: A review. Food Rev. Int. 2016; 32(2): 117-160.

[77] Nazzaro F, Fratianni F, Coppola R. Quorum sensing and phytochemicals. Int. J. Mol. Sci. 2013; 14(6): 1260712619.

[78] Kalia M, Yadav VK, Singh PK, et al. Effect of cinnamon oil on quorum sensing-controlled virulence factors and biofilm formation in Pseudomonas aeruginosa. PLoS One. 2015; 10(8): 1-18.

[79] Oliveira BDÁ, Rodrigues AC, Cardoso BMI, et al. Antioxidant, antimicrobial and anti-quorum sensing activities of Rubus rosaefolius phenolic extract. Ind. Crops Prod. 2016; 84: 59-66.

[80] Sharifi A, Mohammadzadeh A, Zahraei Salehi T, Mahmoodi P. Antibacterial, antibiofilm and antiquorum sensing effects of Thymus daenensis and Satureja hortensis essential oils against Staphylococcus aureus isolates. J. Appl. Microbiol. 2018; 124(2): 379-388.

[81] Moradi F, Hadi N, Bazargani A. Evaluation of Quorum Sensing inhibitory effects of the extracts of Three traditional Medicine plants with Known antibacterial Properties. New Microbes New Infect. 2020: 100769.

[82] Musthafa KS, Ravi AV, Annapoorani A, et al. Evaluation of anti-quorum-sensing activity of edible plants and fruits through inhibition of the n-acyl-homoserine lactone system in Chromobacterium violaceum and Pseudomonas aeruginosa. Chemotherapy 2010; 56(4): 333-339.

[83] Reverchon S, Chantegrel B, Deshayes C, et al. New synthetic analogues of N-acyl homoserine lactones as agonists or antagonists of transcriptional regulators involved in bacterial quorum sensing. Bioorganic Med. Chem. Lett. 2002; 12(8): 1153-1157.

[84] Dickey SW, Cheung GYC, Otto M. Different drugs for bad bugs: antivirulence strategies in the age of antibiotic resistance. Nat. Rev. Drug Discov. 2017; 16(7): 457-471.

[85] Subhadra B, Hwan Oh M, Hee Choi C. Quorum sensing in <em>Acinetobacter</em>: with special emphasis on antibiotic resistance, biofilm formation and quorum quenching. AIMS Microbiol. 2016; 2(1): 27-41.

[86] Willyard C. The drug-resistant bacteria that pose the greatest health threats. Nature. 2017; 543(7643): 15.

[87] Huang G, Shen X, Gong Y, et al. Antibacterial properties of phage Abp1 endolysin (PlyAB1). BMC Infect. Dis. 2014; 14(1): 1-8.

[88] De Breij A, Riool M, Cordfunke RA, et al. The antimicrobial peptide SAAP-148 combats drug-resistant bacteria and biofilms. 2018.

[89] Harding CM, Hennon SW, Feldman MF. Uncovering the mechanisms of Acinetobacter baumannii virulence. Nat. Rev. Microbiol. 2018; 16(2): 91-102.

[90] Zhao X, Yu Z, Ding T. Quorum-sensing regulation of antimicrobial resistance in bacteria. Microorganisms. 2020; 8(3): 1-21.

[91] Costerton JW, Stewart PS, Greenberg EP. Bacterial biofilms: a common cause of persistent infections. Science (80.). 1999; 284(5418): 1318-1322. 
[92] Alves S, Duarte A, Sousa S, Domingues FC. Study of the major essential oil compounds of Coriandrum sativum against Acinetobacter baumannii and the effect of linalool on adhesion, biofilms and quorum sensing. Biofouling. 2016; 32(2): 155-165.

[93] Luís Â, Duarte A, Gominho J, et al. Chemical composition, antioxidant, antibacterial and anti-quorum sensing activities of Eucalyptus globulus and Eucalyptus radiata essential oils. Ind. Crops Prod. 2016; 79: 274-282.

[94] Bhargava N, Singh SP, Sharma A, et al. Attenuation of quorum sensing-mediated virulence of Acinetobacter baumannii by Glycyrrhiza glabra flavonoids. 2015; 10: 1953-1968.

[95] Alibi S, Ben Selma W, Ramos-Vivas J, et al. Anti-oxidant, antibacterial, anti-biofilm, and anti-quorum sensing activities of four essential oils against multidrug-resistant bacterial clinical isolates. Curr. Res. Transl. Med. 2020; 68(2): 59-66.

[96] Paul Bhattacharya S, Mitra A, Bhattacharya A, Sen A. Quorum quenching activity of pentacyclic triterpenoids leads to inhibition of biofilm formation by Acinetobacter baumannii. Biofouling. 2020; 36(8): 922-937.

[97] Mayer C, Muras A, Parga A, et al. Quorum Sensing as a Target for Controlling Surface Associated Motility and Biofilm Formation in Acinetobacter baumannii ATCC® 17978TM. Front. Microbiol. 2020; 11(September): 1-13.

[98] Alam P, Alqahtani AS, Mabood Husain F, et al. Siphonocholin isolated from red sea sponge Siphonochalina siphonella attenuates quorum sensing controlled virulence and biofilm formation. Saudi Pharm. J. 2020; 28(11): 1383-1391.

[99] Saroj SD, Rather PN. Streptomycin inhibits quorum sensing in Acinetobacter baumannii. Antimicrob. Agents Chemother. 2013; 57(4): 1926-1929.

[100] Yang G, Cheng C, Xu GB, et al. Synthesis and antibiofilm evaluation of 3-hydroxy-2,3-dihydroquinazolin-4(1H)one derivatives against opportunistic pathogen Acinetobacter baumannii. Bioorganic Med. Chem. 2020; 28(16): 115606.

[101] Chan K-G, Atkinson S, Mathee K, et al. Characterization of $\mathrm{N}$-acylhomoserine lactone-degrading bacteria associated with the Zingiber officinale (ginger) rhizosphere: Co-existence of quorum quenching and quorum sensing in Acinetobacter and Burkholderia. BMC Microbiol. 2011; 11(1): 51. 